\title{
Point proposal network for reconstructing 3D particle endpoints with subpixel precision in liquid argon time projection chambers
}

\author{
Laura Dominé $\odot,{ }^{3,}{ }^{*}$ Pierre Côte de Soux,${ }^{2}$ François Drielsma, ${ }^{1}$ Dae Heun Koh, ${ }^{3}$ Ran Itay, ${ }^{1}$ \\ Qing Lin, ${ }^{1}$ Kazuhiro Terao, ${ }^{1}$ Ka Vang Tsang, ${ }^{1}$ and Tracy L. Usher ${ }^{1}$ \\ (on behalf of the DeepLearnPhysics Collaboration) \\ ${ }^{1}$ SLAC National Accelerator Laboratory, Menlo Park, California 94025, USA
${ }^{2}$ ICME, Stanford University, Stanford, California 94305, USA
${ }^{3}$ Stanford University, Stanford, California 94305, USA
}

(Received 15 July 2020; accepted 13 July 2021; published 18 August 2021)

\begin{abstract}
Liquid argon time projection chambers (LArTPCs) are particle imaging detectors recording 2D or 3D images of trajectories of charged particles. Identifying points of interest in these images, namely, the initial and terminal points of track-like particle trajectories such as muons and protons, and the initial points of electromagnetic shower-like particle trajectories such as electrons and gamma rays, is a crucial step in identifying and analyzing these particles and impacts the inference of physics signals such as neutrino interaction. The Point Proposal Network is designed to discover these specific points of interest. The algorithm predicts with a subvoxel precision their spatial location, and also determines the category of the identified points of interest. Using as a benchmark the PILArNet public LArTPC data sample in which the voxel resolution is $3 \mathrm{~mm} /$ voxel, our algorithm successfully predicts $96.8 \%$ and $97.8 \%$ of 3D points within a distance of 3 and 10 voxels from the provided true point locations, respectively. For the predicted $3 \mathrm{D}$ points within 3 voxels of the closest true point locations, the median distance is found to be 0.25 voxels, achieving subvoxel-level precision. In addition, we report our analysis of the mistakes where our algorithm prediction differs from the provided true point positions by more than 10 voxels. Among the mistakes we visually scanned 50 events: 25 were due to the definition of true position location, 15 were legitimate mistakes where a physicist cannot visually disagree with the algorithm's prediction, and 10 were genuine mistakes that we wish to improve in the future. Further, using these predicted points, we demonstrate a simple algorithm to cluster 3D voxels into individual track-like particle trajectories with a clustering efficiency, purity, and adjusted Rand index of 96\%, 93\%, and 91\%, respectively.
\end{abstract}

DOI: 10.1103/PhysRevD.104.032004

\section{INTRODUCTION}

Accelerator-based neutrino oscillation experiments have successfully deployed deep convolutional neural networks (CNNs) in their data analysis pipeline [1-3]. Many of the present and future experiments utilize a liquid argon time projection chamber (LArTPC), a class of particle imaging detectors which produce $2 \mathrm{D}$ or $3 \mathrm{D}$ images over many meters of detected charged particle trajectories, with a resolution of the order of mm/pixel. Examples of such experiments along with their respective active volumes

\footnotetext{
*1domine@stanford.edu
}

Published by the American Physical Society under the terms of the Creative Commons Attribution 4.0 International license. Further distribution of this work must maintain attribution to the author(s) and the published article's title, journal citation, and DOI. Funded by SCOAP. include MicroBooNE (90 tons) [4], the Short Baseline Near Detector (SBND, 112 tons) [5], ICARUS (600 tons) [6], and the Deep Underground Neutrino Experiment (DUNE, 40000 tons) [7].

This paper along with Refs. [8,9] are intended to demonstrate the performance of different stages in an end-to-end, machine-learning-based data reconstruction chain for LArTPC experiments [10]. For example, identifying the interaction vertex is at the core of identifying and reconstructing a neutrino interaction in a LArTPC detector: the Point Proposal Network (PPN) significantly improves the efficiency of finding candidate interaction vertices, and hence candidate neutrino interactions, compared to the traditional approach. The output of the chain is a comprehensive description of a LArTPC event which may be used for high-level physics inference. In the context of the DUNE, ICARUS, and SBND experiments (among others), we plan to apply it to neutrino physics analysis such as $\nu_{e}$ appearance and $\nu_{\mu}$ disappearance searches. 
The particle trajectories recorded in LArTPC images often appear as 1D lines in a 2D or 3D space. Their topological features can be diverse, ranging from straight line-like tracks to branching tree-like electromagnetic showers. Points of interest in particle trajectories include the initial point of electromagnetic (EM) particles, referred to as shower-like particles in this paper, as well as the initial and terminal points (earliest and latest along the particle trajectory timeline), collectively referred to as end points, of track-like particles, which include all but shower-like particles. In the process of analyzing an image from the pixel-level energy deposits to build a larger picture of particle trajectories with their respective kinematic properties, detecting these points of interest in the early stage of a data reconstruction chain is critical.

For example, in clustering tasks on EM showers, the initial point can help to define a general direction for the whole shower that includes dozens to hundreds of EM secondaries. This is especially useful for separating neutral pions-a source of major background to the $\nu_{e}$ signal for neutrino oscillation analysis as well as an important sample for detector energy calibration-from cosmic rays and neutrino-nucleus interactions. Finding these points can also be a crucial step in determining a neutrino interaction vertex. If each particle trajectory is associated with these points of interests, the predicted points naturally include candidates for the neutrino interaction vertex. Conventional reconstruction methods exemplified by the Pandora framework [11] identify these points of interest using geometric methods such as sliding linear fits. Their performance is more often reported at the stage of the neutrino vertex identification. For example, in Ref. [12] where Pandora was applied to simulated MicroBooNE events (including detector effects), $68 \%$ of events have a displacement between the true and reconstructed neutrino interaction vertex below $0.74 \mathrm{~cm}$. In our data set we found that $96.8 \%$ of the predicted 3D points are within 3 voxels (i.e., $0.9 \mathrm{~cm}$ ) from the true point locations, which is promising for the future neutrino interaction vertex resolution of our full reconstruction chain.

Localizing an arbitrary number of such points in an image is analogous to a task called object detection in the field of computer vision. Many object detection algorithms based on CNNs have been proposed [13-16], including faster region convolutional neural network (Faster R-CNN) which has been one of the most popular choices for object detection applications and also successfully applied in LArTPC image data [1]. Faster R-CNN consists of a feature extractor $\mathrm{CNN}$ and an attention mechanism called a region proposal network (RPN). The feature extractor consists of convolution layers and pooling layers, and generates a data tensor with low spatial resolution compared to the input. The RPN takes this data tensor and generates region proposals, typically rectangular-shaped bounding boxes, that are likely to contain a target object in the original image resolution. The insight of RPN is to act on a spatially contracted data tensor which contains fewer pixels compared to the original input, thus addressing the challenge of long computing time. R-CNN is a family of algorithms that employ the RPN concept. One of the most recent of these is mask R-CNN [13], which is undeniably the most popular object instance detection algorithm to date.

Inspired by the concept behind RPN, we have designed the Point Proposal Network to identify the previously defined points of interest in a LArTPC image. While RPN is responsible for predicting both the location and size of a bounding box for an object detection, PPN is simplified to propose only the location as the target is a point, not an object. Our goal is to integrate PPN into a generic, full 3D data reconstruction chain for LArTPCs, which consists of multitask deep neural networks, such that the whole chain can be optimized end to end. Building on the previous effort, we use U-ResNet [17] as the feature extractor and implement PPN to predict the position and semantic type of an arbitrary number of points in an image with voxel-level precision.

The contributions of this paper are twofold:

(1) Introduce PPN for reconstructing the 3D end points of track-like particles and the initial point of showerlike particles with subvoxel precision.

(2) Provide a performance benchmark on a public LArTPC simulation data set (PILArNet) for future reference and comparison against other methods.

While in this paper our target is 3D LArTPC images, the concept of PPN is applicable to both 2D and 3D images [18]. Section II introduces the architecture of the U-ResNet network that we use as the backbone for PPN, as well as the details on PPN architecture, the loss definitions, and postprocessing methods. Section III outlines our experimental setup, including details on the public LArTPC data sample that we use. Section IV shows a first benchmark of the PPN performance on this sample.

The study presented in this paper is fully reproducible using a Singularity [19] software container, ${ }^{1}$ implementations are available in the lartpc_mlreco $3 d^{2}$ repository, and public simulation samples [20] are made available by the DeepLearnPhysics Collaboration.

\section{NETWORK ARCHITECTURE}

The network architecture consists of two parts: U-ResNet [17] and PPN. Both blocks include many CNN layers. In order to make our algorithm scalable to a large-scale LArTPC detector analysis, we designed the whole chain using a sparse submanifold convolutional network (SSCN) [21,22].

\footnotetext{
${ }^{1}$ Docker-based image available at https://hub.docker.com/r/ deeplearnphysics/larcv2 under the tag ub18.04-gpu-ana0ml-larcv2.

${ }^{2}$ https://github.com/DeepLearnPhysics/lartpc_mlreco3d.
} 


\section{A. U-ResNet: Feature extractor}

U-ResNet is designed for a voxel-level classification task, called semantic segmentation, for 3D LArTPC images [17]. The architecture of U-ResNet can be divided into two parts, namely, the encoder and decoder. The encoder consists of repeated blocks of convolution and strided convolution layers which down-samples the image resolution while increasing the features dimension, thus learning from key features in an image at different scales. We refer to the number of down-sampling operations as depth. The decoder takes the low-spatial-size, highly compressed data tensor from the encoder and up-samples it back to the original image resolution. After each up-sampling operation, the data tensor of matching spatial size is taken from the encoder output and concatenated to the up-sampled tensor before the combined tensor is further processed by convolution layers in the decoder. The key concept behind the concatenation operation, introduced by the original U-Net authors [23], is to recover the lost spatial resolution information in the encoder block due to strided convolution layers and effectively combine with the abstract features contained in the up-sampled tensor. Convolution layers in the decoder block are trained to best combine high spatial resolution information and abstract feature information. As a result, they learn how to best spatially interpolate abstract features extracted by the encoder back to the original image resolution. Figure 1 shows the architecture of U-ResNet. For the study carried out in this paper, we set the depth of U-ResNet to 6 and used 16 filters at the first convolution layer. The number of filters increases linearly with the depth, and is 96 at the deepest layer.

\section{B. PPN layers}

Within the U-shaped network architecture (see Fig. 1), we implement PPN by introducing additional convolution layers at different spatial resolutions, starting with the most contracted data tensor at the lowest spatial resolution. While these PPN layers could be attached to either the
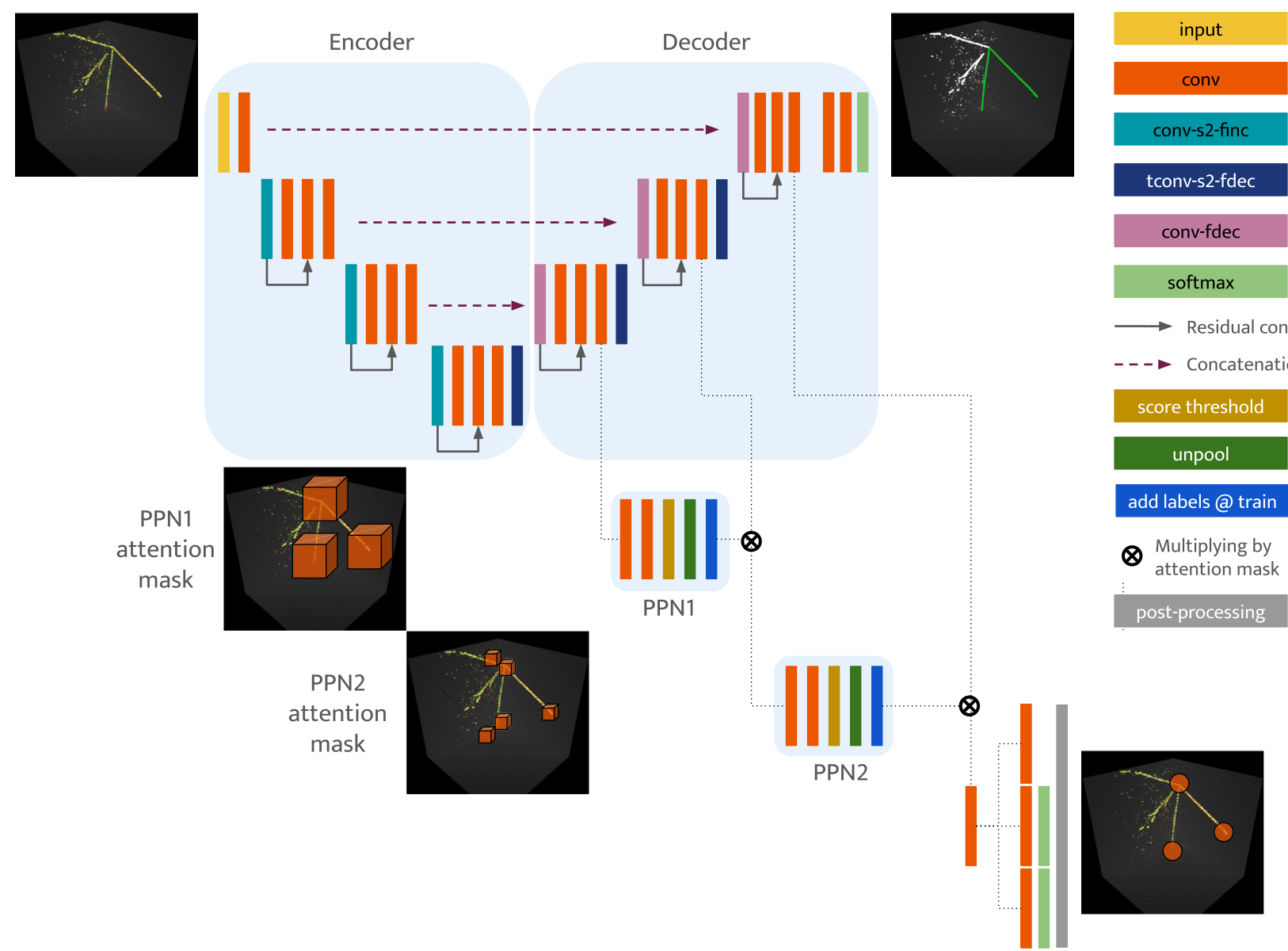

FIG. 1. U-ResNet architecture for semantic segmentation. In this example we say that the U-ResNet has a depth of 3 since we perform three down-samplings. Turquoise boxes represent convolutions with stride 2 that increase the number of filters. Dark blue boxes are transpose convolutions with stride 2 that decrease the number of filters. Purple boxes are convolutions with stride 1 that decrease the number of filters. Blue boxes ("add labels @ train") represent the inclusion of true voxels in the mask of positive voxels, and only apply during training. The gold boxes are a score thresholding $(>0.5)$ operation on the softmax of predicted scores. The spatial size of the data tensor is constant across the horizontal dimension. 
encoder or the decoder of U-ResNet, it is more powerful to attach them to the decoder block as data tensors generated by the decoder should be more information rich. At the deepest level and coarsest spatial resolution, the so-called PPN1 produces a softmax score of a value between 0 and 1 for each voxel, which indicates whether or not the voxel contains the location coordinate of any of the true points, i.e., the target $3 \mathrm{D}$ points to be detected. We call this the detection score in the following. We call the voxels positive if the detection score is above the set threshold value. We call other voxels negative. Positive voxels yield an attention mask that we can use at the next step. At an intermediate level and medium spatial resolution, we up-sample the mask predicted by PPN1 and use it as an attention mask to preselect candidate positive voxels. The so-called PPN2 layer then similarly predicts a subset of positive voxels among these preselected voxels in the attention mask at this spatial resolution. Finally, we up-sample the result of PPN2 to the original image resolution and use it as another attention mask. The final layer, the so-called PPN3, is made of $3 \times 3$ convolutions which predict the following quantities for each voxel that has been selected through these successive attention masks:

(1) a detection score (of being a voxel within some neighborhood of a ground true point, for which we choose a distance threshold of 5 voxels),

(2) a 3D position (offset with respect to the voxel center), and

(3) a type score (for the point to belong to a semantic type).

\section{Loss definitions}

Among all voxels $\overrightarrow{x_{i}}$ we define true voxels $A$ as voxels within a certain distance threshold $d_{\text {positive }}$ from the true points $\overrightarrow{q_{j}}$, and all other voxels as negative:

$$
A=\left\{1 \leq i \leq N \mid \exists j\left\|\overrightarrow{x_{i}}-\overrightarrow{q_{j}}\right\|<d_{\text {true }}\right\},
$$

where $N$ is the number of voxels in the input data tensor at a certain PPN layer.

We define several losses. First, for all input voxels, we compute a cross-entropy loss for a positive/negative classification task at each PPN- $i$ layer and then average over all voxels. For $i=1,2,3$, if $\vec{y}_{k} \in\{0 ; 1\}$ indicates whether the voxel is positive or negative in the labels and $p_{k}$ is the predicted softmax score for this voxel to be positive,

$$
\mathcal{L}_{\text {detection }}^{i}=-\frac{1}{N_{i}} \sum_{k=1}^{N_{i}} y_{k} \log \left(p_{k}\right)+\left(1-y_{k}\right) \log \left(1-p_{k}\right) .
$$

Second, only on true voxels, we define a linear distance loss on the predicted positions. We consider the distance to the closest true point $\vec{q}$. The raw predictions $\vec{p}$ of the network are actually shifts with respect to the center of the subject voxels $(0.5+\vec{x})$ :

$$
\mathcal{L}_{\text {distance }}=\frac{1}{N_{3}} \sum_{i=1}^{N_{3}} \mathbb{1}_{A} \min _{j}\left\|\vec{p}_{i}+0.5+\vec{x}_{i}-\vec{q}_{j}\right\| .
$$

Third, only on true voxels, we compute a cross-entropy loss for a point-type prediction. The predicted point type is compared with the semantic type of the closest true point. If $N_{c}$ is the total number of semantic types for a point, $\vec{y}$ is a one-hot encoded vector indicating to which type the point belongs, and $p_{c}$ is the predicted probability that the point belongs to a semantic type $c$, then

$$
\mathcal{L}_{\text {type }}=-\frac{1}{N_{3}} \sum_{i=1}^{N_{3}} \mathbb{1}_{A} \sum_{j=1}^{N_{c}} y_{c} \log \left(p_{c}\right) .
$$

Finally, the sum of all losses is minimized:

$$
\mathcal{L}=\mathcal{L}_{\text {type }}+\mathcal{L}_{\text {distance }}+\sum_{i=1,2,3} \mathcal{L}_{\text {detection }, i} .
$$

\section{Post-processing}

The architecture that we proposed so far will yield a prediction of a position, detection score, and semantic-type score for each voxel that has been selected in the last layer at the original image resolution. The number of such positive voxels whose predictions are considered is related to the attention mask predicted by PPN2 and the spatial size ratio between the PPN2 layer and the original image size. This will dictate for each voxel predicted as positive at the PPN2 level how many voxels are selected at the last layer. Hence, we might have many proposals whose positions are clustered near a true point, with different scores and type predictions. We need a strategy to combine overlapping proposals to deduce the candidate of distinct 3D points, and we want this strategy to value both accurate positions and type predictions. In this paper, we adopt the following simple post-processing scheme.

(1) Thresholding on the detection scores; for example, we require a score value of 0.5 or higher to be considered positive.

(2) We then run the DBSCAN [24] clustering algorithm on the positive point positions in order to identify clusters of PPN proposals that overlap each other, presumably near the target end points. The hyperparameters of DBSCAN are set to $\epsilon=1.99$ in voxel units for the maximum Cartesian distance $\epsilon$ between two points to be clustered together, and min_samples $=1$ for the minimal point count in a cluster. $\epsilon$ must be small enough to avoid merging together predicted end points of short tracks.

(3) Pooling operation on the points that belong to the same cluster in order to deduce a single score, type predictions, and 3D position: we use average pooling for the 3D coordinate locations, and maximum 

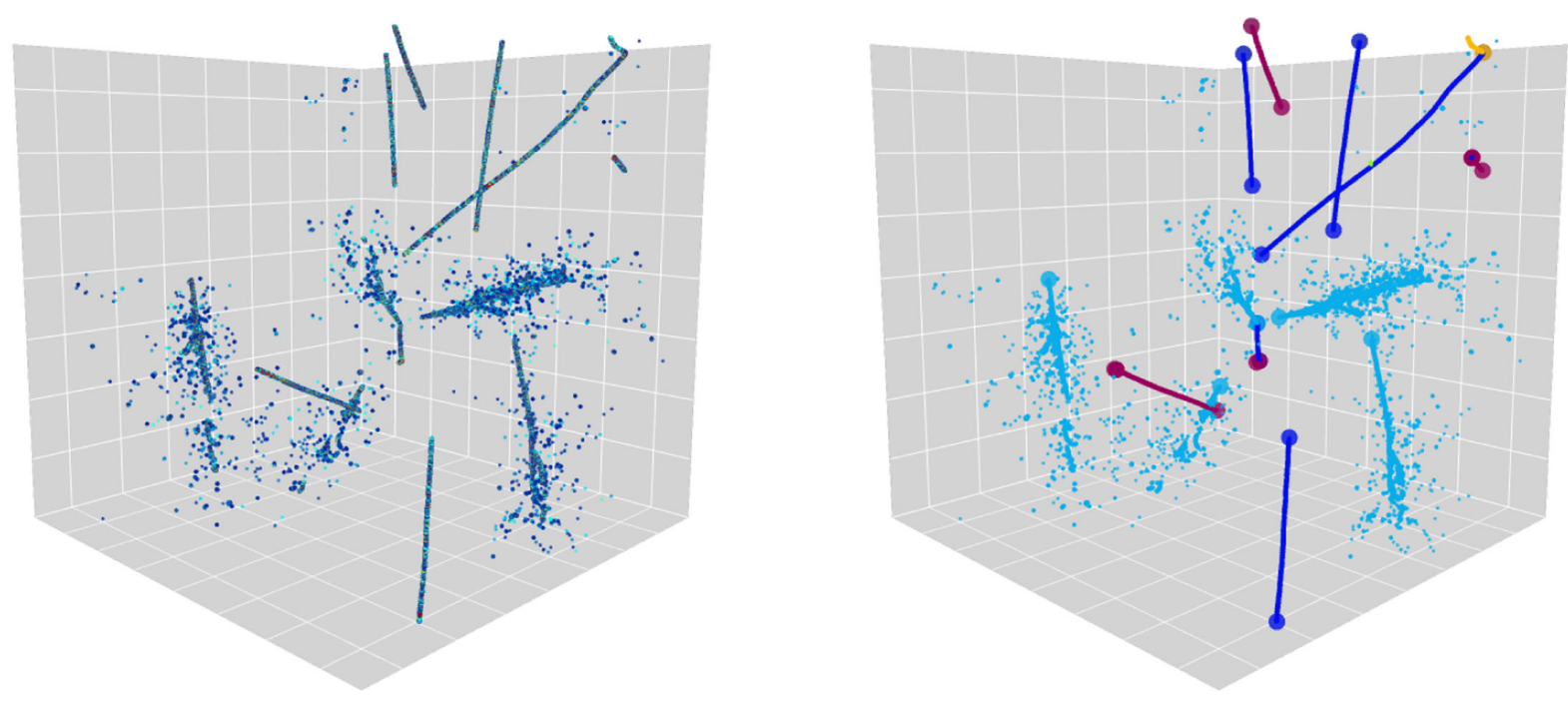

FIG. 2. Simulated LArTPC event from our data set. The left picture (network input) shows energy deposits from charged particle trajectories, whose color corresponds to an energy scale. In the right image (labels), each voxel is assigned one of five colors: HIPs in purple, MIPs in dark blue, electromagnetic showers in light blue, delta rays in green, and Michel electrons in orange.

pooling for the scores including the positiveness prediction and individual semantic type.

(4) Finally, we enforce that a point detected by PPN as a type among $c_{i}$ (set of types, with type score $>0.5$ for each type $c_{i}$ ) needs to be within 2 voxels of a voxel predicted by U-ResNet to have one of the $c_{i}$ types.

\section{EXPERIMENTS}

\section{A. Data set}

We use 3D LArTPC particle images from the PILArNet data set [20], an open data set made available by the DeepLearnPhysics Collaboration. ${ }^{3}$ While lacking detectorspecific effects, it includes a voxel-wise spatial smearing of the charge in order to mimic a real detector resolution. The PILArNet images are thus representative of the geometrical and basic calorimetric features of particle trajectories in a LArTPC detector. The application of this work to realistic LArTPC data sets-which would include among others detector effects, gaps in tracks, recombination effects, and artifacts due to the 2D to $3 \mathrm{D}$ image reconstructionrequires separate, experiment-specific studies. In this paper, our algorithm is optimized using a supervised training method, which may depend on the accuracy of physics modeling (i.e., simulation). More work on machine learning techniques addressing such challenges may follow.

We use the largest 3D image size in the data set, a cubic volume with each side 768 voxels (453 million voxels) at $3 \mathrm{~mm} /$ voxel spatial resolution. Figure 2 shows an example image from this data set. The data set contains 80000 images for the training set and 20000 images for the test set

\footnotetext{
${ }^{3}$ https://dx.doi.org/10.17605/OSF.IO/VRUZP.
}

where each image contains several particles traversing the LAr volume. The PILArNet provides five types for the voxel-level semantic category. These include heavily ionizing particles (HIPs, e.g., protons), minimum ionizing particles (MIPs, e.g., muons and pions), EM showers, delta rays, and Michel electrons from muon decays. Further, the data set provides particle-level metadata including end points of HIP and MIP particles as well as the initial point of other particle types including EM showers, delta rays, and Michel electrons. These 3D points are provided with a floating-point precision in the unit of voxels, and used as true points for training PPN. More details can be found in the PILArNet reference [20].

\section{B. Training details}

We drop the point labels for particles with less than $10 \mathrm{MeV}$ in total energy deposit or a total voxel count of less than 7 voxels, which corresponds to a trajectory of $7^{1 / 3} \sim$ 1.9 voxels $\sim 0.6 \mathrm{~cm}$ in length, as a typical trajectory width is a few voxels wide in each transverse spatial direction. This residual range would correspond to a proton of kinetic energy 20-25 MeV, a low threshold for LArTPC. The PPN1 and PPN2 layers have a spatial size of 24 and 96 voxels, respectively. During training, we add true voxels to the attention masks generated by the PPN1 and PPN2 layers so that the subsequent layers, namely, PPN2 and PPN3, can be trained with some mixtures of true and false voxels. This allows all PPN layers to train simultaneously from the beginning.

The batch size is 64 and we used an Adam optimizer with learning rate 0.001 to train the network. Training the U-ResNet alone first for 20000 iterations, then U-ResNet + PPN for another 20000 iterations, took 184 hours on an Nvidia V-100 GPU for the total of 32 epochs. PPN might 
have less parameter phase space available if it starts learning after U-ResNet feature maps are already highly specialized, and it might have to train longer. On the other hand, the whole network (i.e., U-ResNet + PPN) can be trained from scratch without having to separate into two stages, in which case 40000 iterations took 231 hours. However, since PPN relies on the features of U-ResNet, its performance is expected to be poor until the U-ResNet stage reaches sufficient accuracy, which in turn might slow down the speed of U-ResNet learning. Unless stipulated otherwise, the default configuration for the rest of this paper is the two-stage training.

\section{RESULTS}

\section{A. Position precision}

Figure 3 is a visual example of predictions made by U-ResNet + PPN. Figure 4 shows the distribution of distance from a true point to the closest predicted point. For all true points, $95.1 \%$ and $97.8 \%$ of them are within a voxel distance of 3 and 10 from a predicted point. The figure also shows the distribution of distances from a predicted point to the closest true point, and we find that our algorithm successfully predicts $96.8 \%$ and $97.8 \%$ of 3D points within the voxel distance of 3 and 10 from the true points, respectively. If we look at semantic-type-wise results, we find that the fraction of true points which are more than 3 voxels away from any predicted point is $7 \%$, $2.1 \%, 8.2 \%$, and $1.6 \%$ for the HIP, MIP, EM shower, and Michel electron types, respectively. For this analysis and Fig. 4, we excluded delta-ray-type true and predicted points since the true point coordinates for delta rays provided in PILArNet are less precise than those of MIP, HIP, EM shower, and Michel electron types. This is likely because

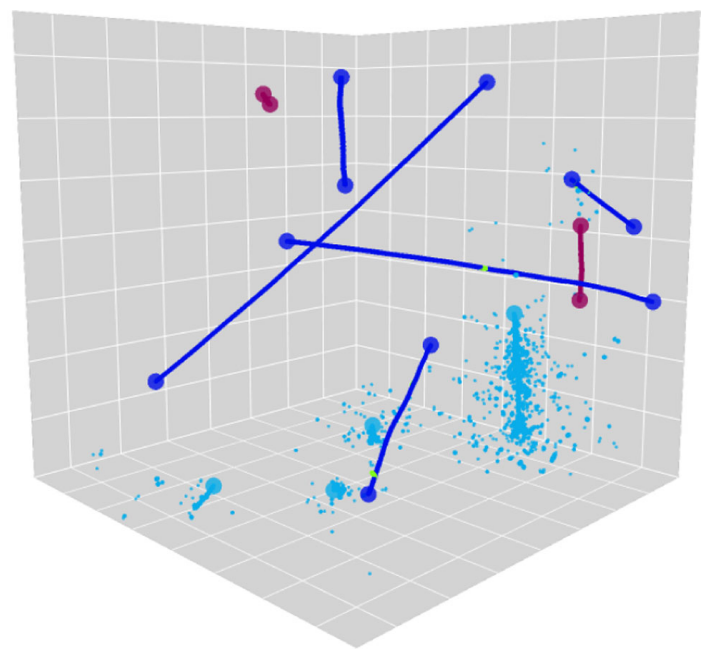

FIG. 3. Example of predictions by U-ResNet + PPN. The voxels' color corresponds to their semantic class as predicted by U-ResNet. The dots are proposed by PPN, and the dots' color represents the point type predicted by PPN. See Fig. 2 for the color legend. the initial points of delta rays often overlap with a muon trajectory, which typically has a width of a few voxels or more. We considered the predicted points to be delta-ray type if the point has a delta-ray-type score of 0.5 or higher.

For those predicted points within 3 voxels of the closest true point, the median distance between the positions of a predicted point and the closest true point is measured to be 0.25 voxels. If PPN is only sensitive to identify a true voxel in which a true point is present, and if it is not capable of regressing the position at the subpixel level, we expect this resolution to be 0.66 , which is the median distance between two random point positions in a voxel. We note that $17 \%$ of the true points provided by PILArNet are located exactly at the center of a voxel, which is typically observed as a result of an end-point approximation within the recorded volume when a particle is exiting or entering the volume. For other true points whose position is not fixed exactly at the voxel center, the correlation of the distance between a predicted point to the closest true point and the displacement of that predicted point from the true voxel center is shown in Fig. 5. When PPN predicts a point within the correct true voxel, geometrically the distance from the voxel center to the predicted point must be between 0 and 0.866 . The two distances show almost no correlation in this range, which shows that PPN position resolution is uniform and independent of the true point location within the true voxel. This demonstrates that our algorithm achieved subvoxel-level precision for this reconstruction task.

\section{B. Type prediction accuracy}

Before evaluating the point-type prediction performance, it is useful to remind ourselves of the performance of U-ResNet. In this particular training, the confusion matrix that we obtain is shown in Fig. 6. We can then look at the distance from predicted points to true voxels of a certain

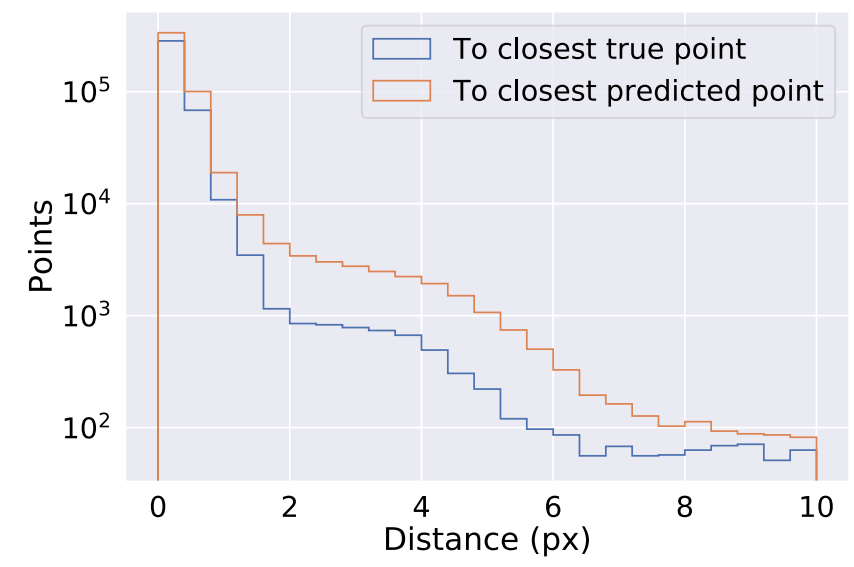

FIG. 4. Distance from true points to the closest predicted points, and from predicted points to the closest true points. Both the true and predicted points of delta-ray type are excluded in this plot. $97.8 \%$ of the points are contained in the $x$ axis range for both histograms. 


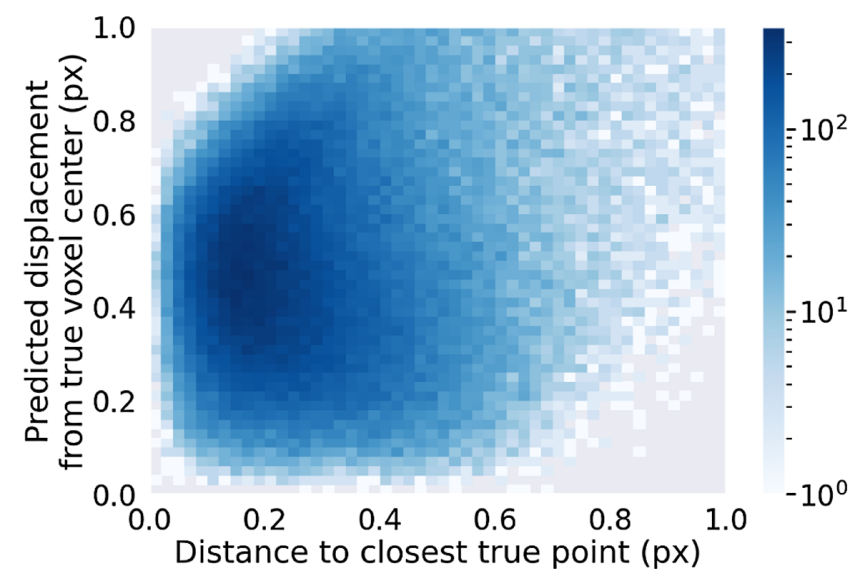

FIG. 5. Correlation between the distance from a predicted point to the closest true point, and the distance from a predicted point to the voxel center of the corresponding true point. We selected predicted points that are within 3 voxels of a true point. About $3.6 \%$ of these predicted points are associated with true points that PILArNet locates exactly at the center of a voxel. They are not pictured here.

semantic type. For example, we expect predicted points with a high Michel- or delta-ray-type score to be close to MIP voxels in the labels, and Fig. 7 confirms this. Figure 8 shows that imposing the maximal distance threshold of 2 voxels between a predicted point with a high type score for a set of types and a voxel whose predicted type matches one of them is reasonable.

We define purity and efficiency metrics for the pointtype prediction as follows: for a given predicted point, we consider all semantic types for which it has a score $>0.5$

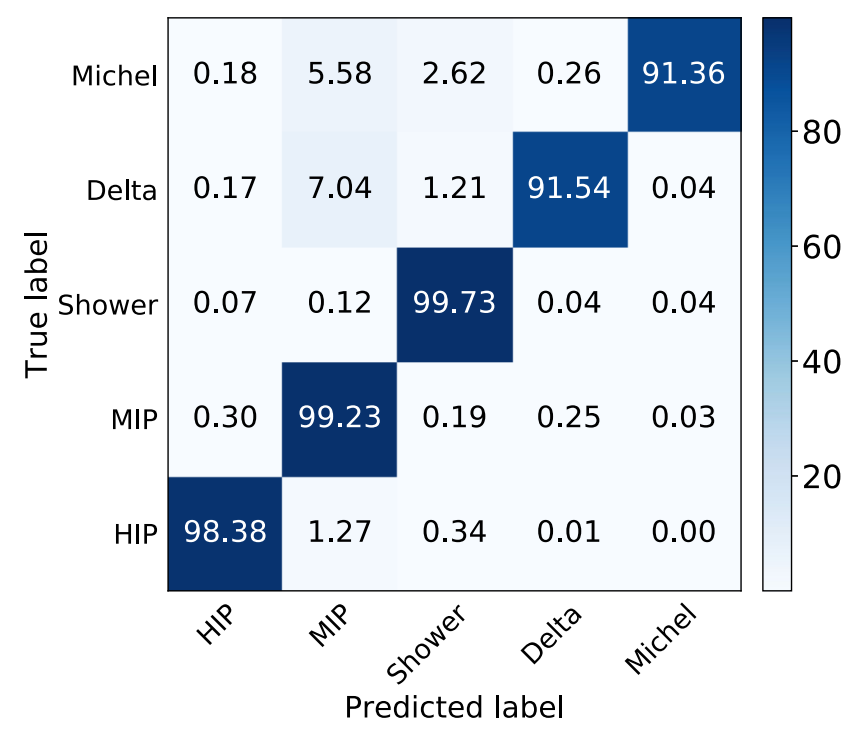

FIG. 6. Confusion matrix for U-ResNet. Each cell contains the fraction of voxels in percent belonging to a certain true semantic type on the vertical axis that have been predicted as the semantic type shown on the horizontal axis. Each row sums to $100 \%$.

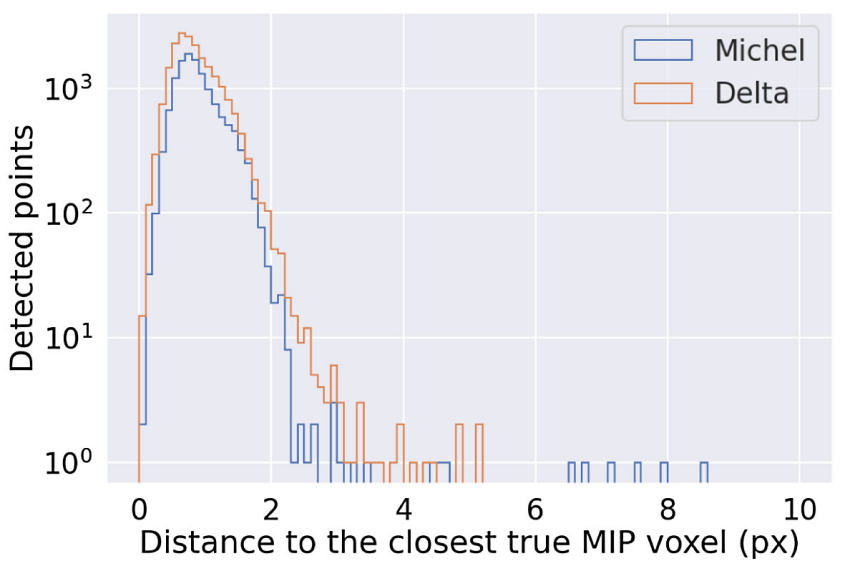

FIG. 7. Looking at the distance between predicted points of Michel electron or delta-ray type (i.e., with a corresponding semantic type score $>0.5$ ) and the closest voxel with the true semantic type of MIP.

and we refer to them as predicted types. We count a predicted type as matched if there exists a true point of the same type within 5 voxels. We note that one point may be associated with multiple types, and one point may contribute as many times as its associated type counts under this scheme. The fraction of matched predicted types is our purity metric. Similarly, for a given true label type, we say that it is matched if there is a predicted point within 5 voxels which has a score $>0.5$ for the same semantic type. The fraction of matched true types is our efficiency metric. Under these definitions, we find a purity of $96.3 \%$ and an efficiency of $89.2 \%$. Table I breaks down these purity and efficiency metrics for each semantic class. The purity is significantly higher than the efficiency, which indicates that while currently predicted types are highly accurate, there is a space for PPN to improve to predict all possible types associated with a predicted point. This may be related to the

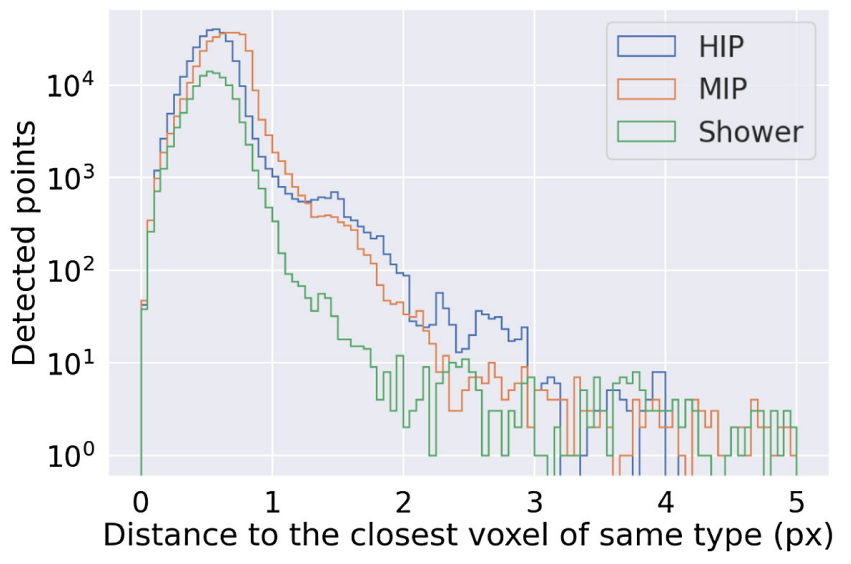

FIG. 8. This histogram shows the distance from a predicted point before post-processing with a type HIP, MIP, or EM shower (i.e., type score $>0.5$ ) to the closest voxel of the same type as predicted by U-ResNet. 1024 events are used in this histogram. 
TABLE I. Purity and efficiency of the point-type predictions for different semantic classes. Purity for a class $\mathrm{X}$ is the fraction of predicted points with a type score $>0.5$ for this class $\mathrm{X}$, and within 5 voxels of a true point whose type matches one of the predicted point types (i.e., type score $>0.5$ ). Efficiency for a class $\mathrm{X}$ is the fraction of true points of type $\mathrm{X}$ within 5 voxels of a predicted point with type score $>0.5$ for this class $\mathrm{X}$.

\begin{tabular}{lcccc}
\hline \hline Class & HIP & MIP & Shower & Michel \\
\hline Purity & $97 \%$ & $98 \%$ & $92.2 \%$ & $99.3 \%$ \\
Efficiency & $85.3 \%$ & $93.4 \%$ & $90.7 \%$ & $89.7 \%$ \\
\hline \hline
\end{tabular}

architecture where PPN is coupled to U-ResNet which ultimately predicts a single type per voxel.

\section{Mistakes analysis}

About $2.2 \%$ of predicted points, excluding points predicted as delta-ray type, are more than 10 voxels away from any true point. Let us call them far mistakes. Among them, $25.8 \%$ have a high ( $>0.5)$ type score of being HIP, $21.9 \%$ for MIP, and $53.8 \%$ for shower. We have visually scanned event displays of these mistakes and report their nature in this section. In summary, we found that a large fraction of far mistakes were due to issues with true points or legitimate mistakes where authors cannot visually distinguish from correct predictions.

\section{Fragmented EM showers}

An EM shower is initiated by an EM particle including an electron, a positron, or a gamma ray, and develops a cascade of them through radiations of gamma rays. In physics analysis, typically a whole cascade is conveniently treated as one EM shower instance instead of identifying dozens to hundreds of secondaries. This is shared in the PILArNet data set where EM shower information, such as the initial point, is provided for the whole cascade. In LArTPC, however, given the average radiation length of $14 \mathrm{~cm}$ [25], which corresponds to 47 voxels, we expect that some radiated gamma rays in the cascade may be separated by significant gaps. This results in cases where a single EM shower may appear indistinguishable from two or more separate, overlapping EM showers.

In those cases, PPN may place multiple initial points within a single shower, as shown for example in Fig. 9. While this may visually appear reasonable, they can be the cause of far mistakes as PILArNet provides only one initial point for the whole shower. Among the far mistakes, more than half $(53.2 \%)$ have a high $(>0.5)$ type score for being EM shower and are within 2 voxels distance from voxels of true shower type in semantic segmentation labels.

\section{Mistakes due to tracks (HIP/MIP)}

$49.4 \%$ of the far mistakes have a HIP or MIP point type score $>0.5$. We randomly sampled 20 cases with one far

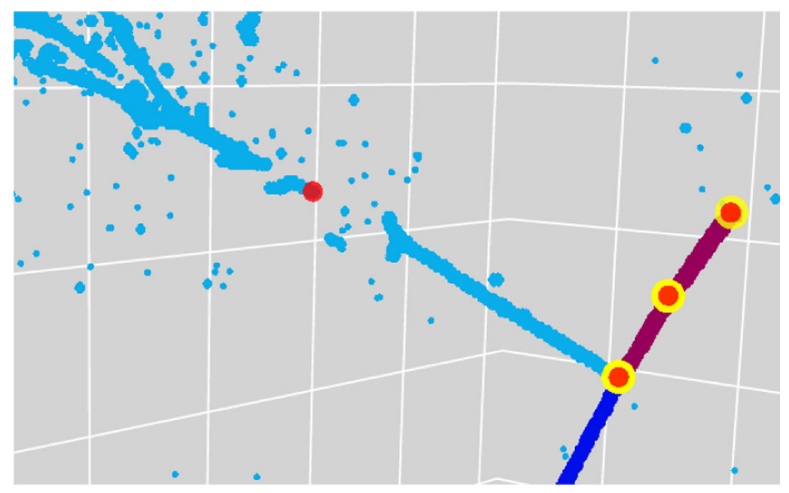

FIG. 9. This picture shows the semantic and point predictions of U-ResNet and PPN. Predicted EM shower voxels are in cyan, MIP in dark blue, and HIP in purple. The yellow points are true points. The red points are predicted by PPN. The shower fragment on the left belongs to the EM shower coming from the right, and PILArNet only provides a single initial point for the whole shower (the yellow dot at the shower start on the right, where PPN correctly predicted another EM shower point).

mistake with a high HIP score. Fifteen of them $(75 \%)$ were due to very small HIP trajectories for which PPN made good predictions but true points were missing. This is caused by the fact that these trajectories fall below the threshold that we impose to define true points ( $>7$ voxels, $>10 \mathrm{MeV}$ total energy deposit), leading to missing true points as shown in Fig. 10. Two out of the remaining five cases were found to be "legitimate mistakes" due to a kink in a trajectory, as shown in Fig. 11. The last three cases were genuine mistakes (e.g., a point predicted in the middle of a trajectory without any obvious kink).

On the other hand, we also sampled 20 events with far mistakes with a high MIP score. One case was due to a short trajectory missing true points, and one was a rare case where PPN made an extra, faulty prediction at the crossing point of two MIP trajectories that accidentally overlapped in the $3 \mathrm{D}$ space. The majority (12 cases) were legitimate

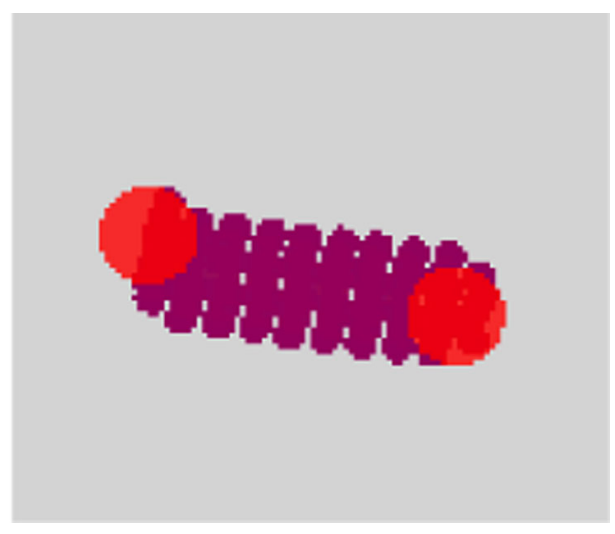

FIG. 10. Example of a short trajectory (purple voxels) that is lacking true points due to small total energy deposit. The red points are predicted by PPN. 


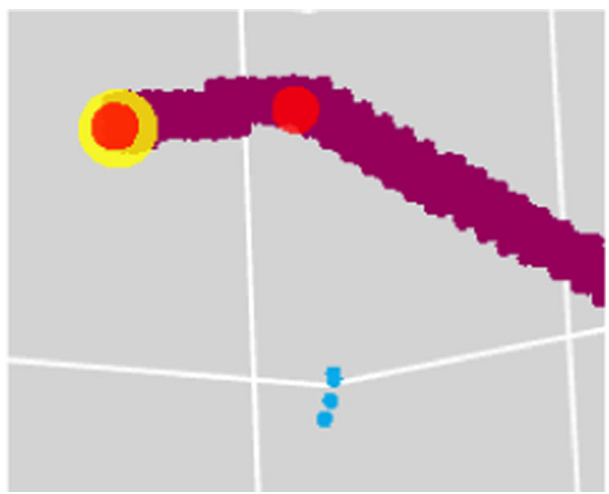

FIG. 11. Example of a kink along a HIP trajectory (purple voxels) causing a legitimate mistake by PPN. The yellow points are true points. The red points are predicted by PPN.

mistakes due to a kink in a trajectory. The rest (six cases) were genuinely bad mistakes.

\section{Trajectories affected by the boundaries}

$10.1 \%$ of far mistakes are within 5 voxels of an image boundary, indicating that they may come from a particle trajectory crossing the image volume boundary. MIP trajectories are more likely to cross a volume boundary due to their length. Hence, they are more affected by boundaries. Among the far mistakes that are more than 5 voxels away from the boundary, only $18 \%$ have a high MIP type score. This fraction increases to $54 \%$ in the region within 5 voxels from the boundary, while negligible statistical change was observed for predicted points of other types.

We have visually scanned ten randomly selected far mistakes of a high MIP type score in this region next to the boundary. One of them was a legitimate mistake due to a kink in a trajectory, similar to the dominant case of MIP far mistakes found and described previously. The rest (nine cases) of the MIP mistakes near the boundary were all due

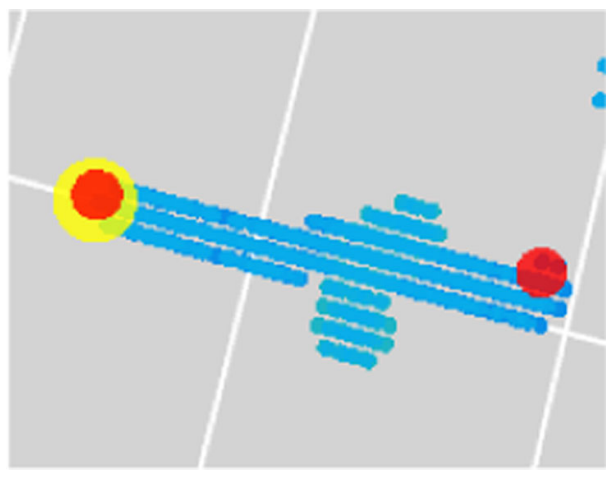

FIG. 12. Example of shower trajectory exiting the volume. All the cyan (true shower) points were mistakenly classified as MIP by U-ResNet. The yellow points are true points. The red points are PPN predictions with high MIP score.

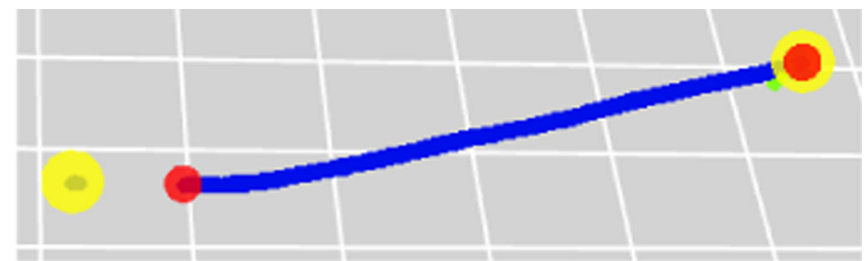

FIG. 13. Example of MIP trajectory exiting and briefly reentering the volume. The yellow points are label points. The red points are PPN prediction.

to issues related to true points. These issues include exiting shower trajectory, which gets classified as MIP by U-ResNet (Fig. 12) and results in a too short trajectory and loss of true points as previously described for HIP cases, a MIP trajectory that exited and reentered the image volume (Fig. 13) for which the true points provided in PILArNet appear unreasonable, and also what appears as a genuine mistake of true point location on the boundary provided by the PILArNet data set (Fig. 14). We conclude therefore that the majority of far mistakes made by PPN are due to either issues related to true points or legitimate mistakes that visually appear reasonable.

\section{Others}

We also compared the PPN performance in two training scenarios: a single-stage training where we start training from scratch both U-ResNet and PPN at the same time for 40000 iterations, and a two-stage training where we train U-ResNet for 20000 iterations first before adding the PPN layer and continue training of U-ResNet + PPN for 20000 more iterations. Everything else is identical between the two schemes. The fraction of true points that are within 10 voxels of a predicted point is $98.2 \%$ and $97.8 \%$, respectively. The fraction of predicted points that are within 10 voxels of a true point is $97.8 \%$ in both cases. The fraction of true points which are more than 3 voxels away from any predicted point is $5.2 \%$ and $5.4 \%$, respectively. There is no significant difference between the two training schemes,

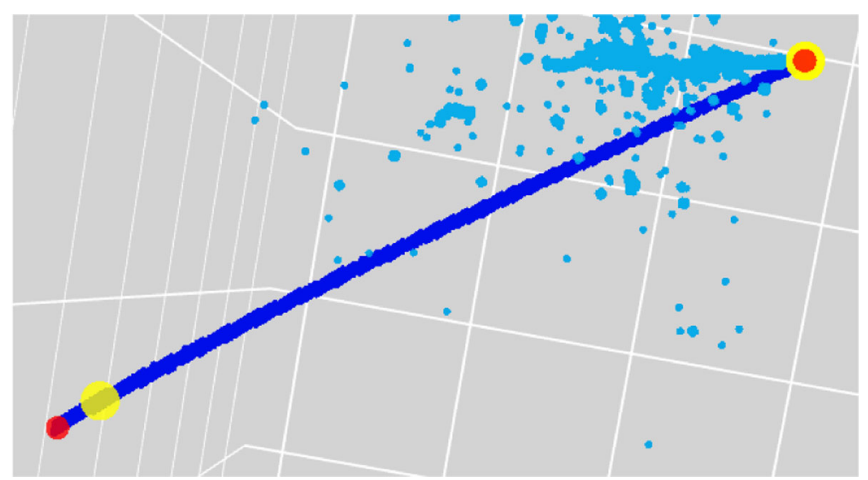

FIG. 14. An example issue of a true point that appears to be mistakenly shifted. The yellow points are true label points. The red points are PPN predictions. 
TABLE II. Resource usage in time and memory of U-ResNet and U-ResNet + PPN architectures, on Nvidia V-100 GPUs.

\begin{tabular}{llllcc}
\hline \hline & \multicolumn{2}{c}{ Duration $(\mathrm{s})$} & & \multicolumn{2}{c}{ Memory $(\mathrm{GB})$} \\
\cline { 2 - 3 } & Train & Test & & Train & Test \\
\hline U-ResNet only & 14.3 & 4.9 & & 9.9 & 2.2 \\
U-ResNet + PPN & 20.5 & 7.6 & & 10.8 & 2.2 \\
\hline \hline
\end{tabular}

which confirms that the PPN learning is conditioned by the U-ResNet performance.

Table II shows that PPN layers have very little impact on memory usage (about $1 \mathrm{~GB}$ at train time, negligible at inference time). However, they are responsible for about $30 \%$ of the total computation time, if compared with the U-ResNet-only resource usage.
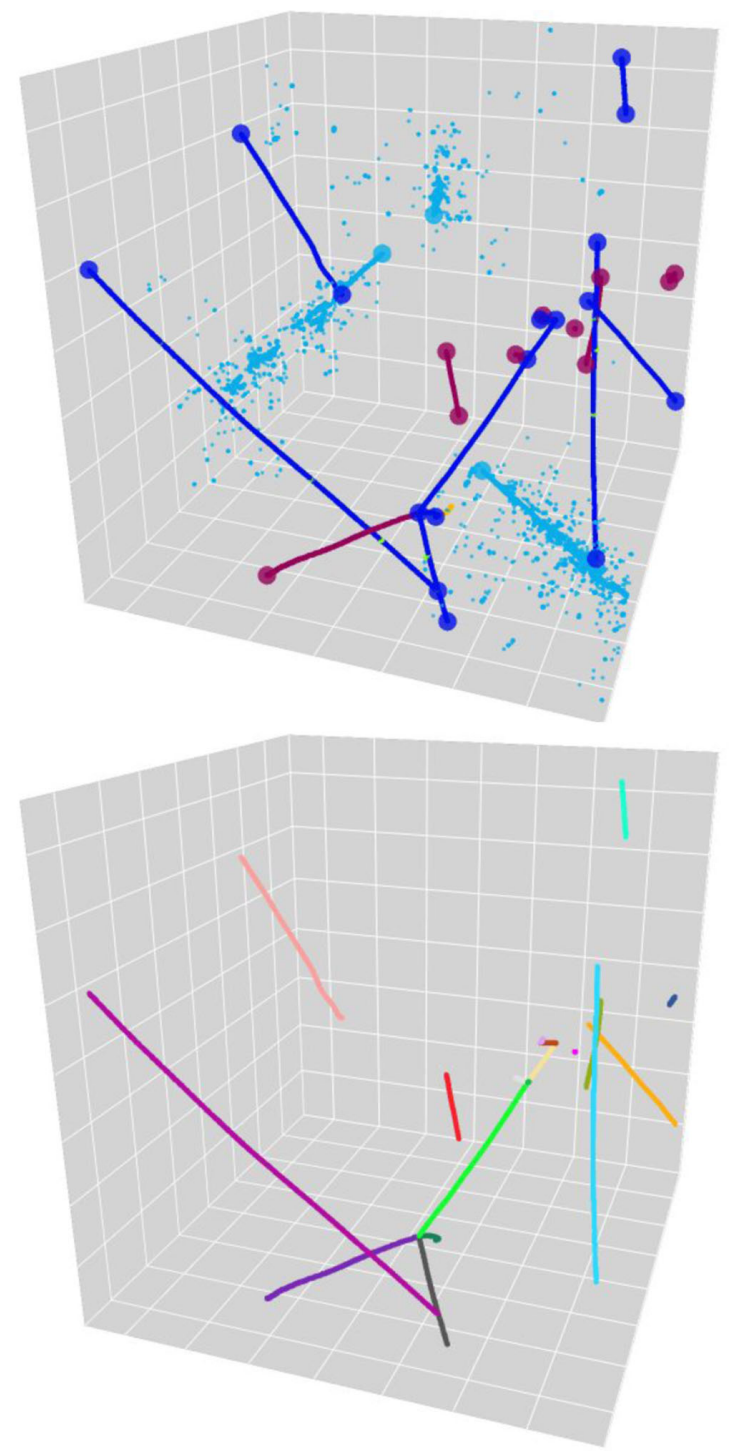

\section{E. Track clustering}

Last, we report a simple application of U-ResNet and PPN for clustering voxels to identify individual track-like particles. This clustering task belongs to the next important step in the LArTPC data reconstruction pipeline. Using the output of U-ResNet and PPN, a very simple clustering algorithm can be designed. First, for each predicted semantic type, we run a density-based clustering algorithm such as DBSCAN [24] on the voxels predicted to belong to track-like particles (i.e., HIP and MIP types). We use here the parameters $\epsilon=4$ and min_samples $=7$ for DBSCAN. This will cluster together particle trajectories that are spatially adjacent, such as tracks coming out of the same interaction vertex. To mitigate this issue we can use the points predicted by PPN to "break" the predicted clusters: for each predicted cluster from
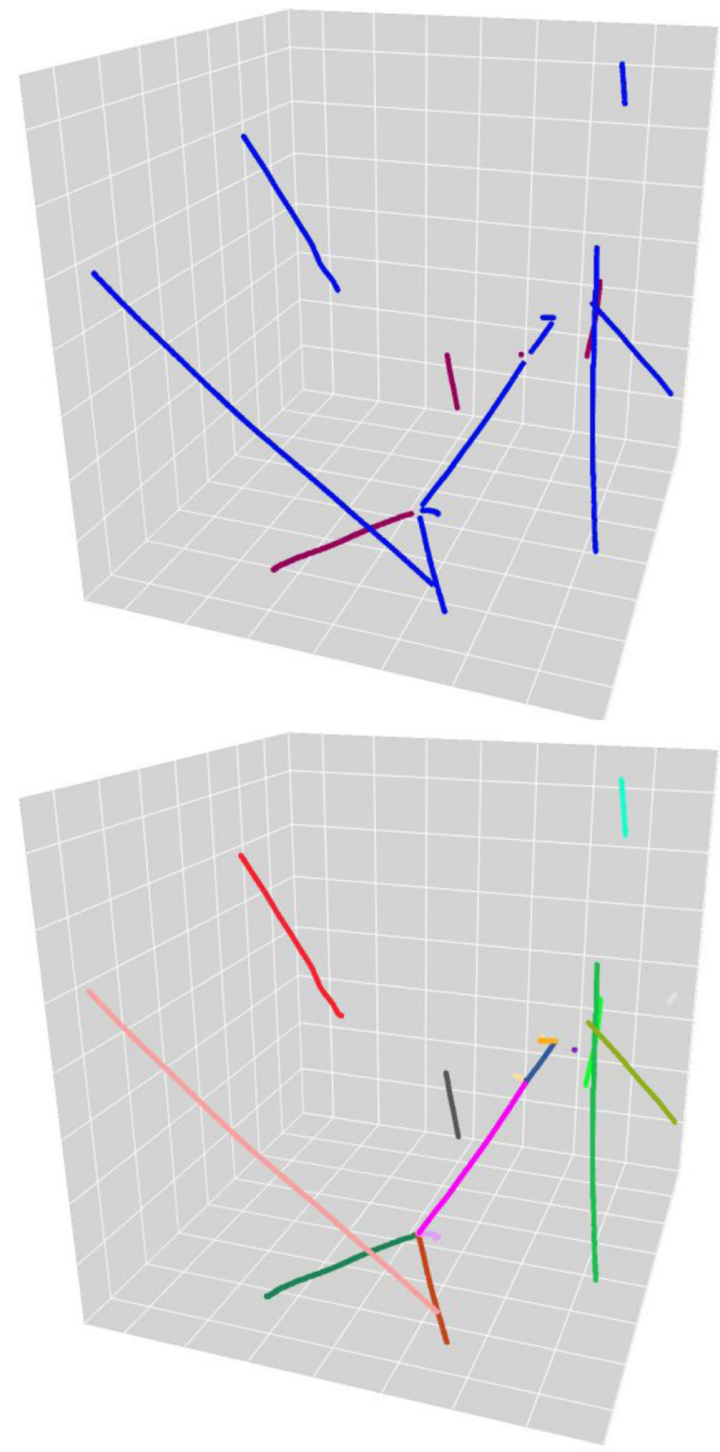

FIG. 15. Track clustering. Top left: U-ResNet + PPN predictions. Top right: selecting U-ResNet track predictions only and removing voxels around PPN predictions. A radius of 10 voxels is used to make the gaps visible in this figure, but the clustering algorithms use a radius of 7 voxels. Bottom left: true track particle clusters. Bottom right: predicted track particle clusters. 
the first step and associated closest predicted points, we mask a sphere of 7 voxels around each predicted point, run DBSCAN again to reconstruct the main trunk of individual track-like particles, and assign the remaining voxels in the masked regions to the closest track-like cluster to complete individual trajectories. Figure 15 illustrates this simple algorithm.

We define metrics of purity and efficiency per cluster, as a fraction of the predicted cluster voxels overlapping with the true cluster and a fraction of the true cluster voxels overlapping with the predicted cluster, respectively. We also look at the adjusted Rand index (ARI) metric [26] per true semantic type (HIP and MIP), averaged over events, to get a sense for the overall clustering performance. We find for efficiency/purity/ARI metrics the values of $0.96 / 0.93 / 0.91$ for track-like clusters.

\section{CONCLUSION}

We have introduced the Point Proposal Network. Building on the previous development of U-ResNet [17], we showed that PPN is capable of detecting the end points of track-like particles as well as the initial point of showerlike particles. PPN successfully predicts $96.8 \%$ and $97.8 \%$ of 3D points within a voxel distance of 3 and 10 (i.e., 0.9 and $3 \mathrm{~cm}$ ) from the true points, respectively. For predicted points within 3 voxels of the closest true point, PPN achieves subvoxel-level precision with a median distance of 0.25 voxels.

A critical step in the reconstruction algorithms of LArTPC detectors for neutrino selections is the localization of the neutrino interaction vertex. When the conventional reconstruction framework Pandora was applied to simulated MicroBooNE events [12], 68\% of events had a displacement between the true and reconstructed neutrino interaction vertex below $0.74 \mathrm{~cm}$. Given that candidates for a neutrino interaction vertex naturally find themselves among PPN predicted points, the PPN performance reported in this paper is highly promising for significantly improving the efficiency of neutrino identification. Standard methods such as that of Ref. [12] also report that the vertex identification is often misled by specific topologies such as a muon decaying into a Michel electron, which makes the downstream end point of the muon look like a strong vertex candidate. The quality of the PPN point-type predictions, for which Michel electrons are a separate semantic class, would allow us to improve on this common mistake.

PPN is also the first benchmark algorithm for PILArNet for reconstructing particle positions. Using the output of U-ResNet and PPN, we demonstrated a simple set of algorithms to cluster 3D voxels into individual tracklike particles. We reported that our algorithms achieved a voxel clustering efficiency/purity/ARI of 0.96/0.93/0.91. U-ResNet and PPN are part of a scalable, deeplearning-based data reconstruction chain for LArTPC detectors [8-10].

\section{ACKNOWLEDGMENTS}

This work is supported by the U.S. Department of Energy, Office of Science, Office of High Energy Physics, and Early Career Research Program under Contract No. DE-AC02-76SF00515.
[1] R. Acciarri et al. (MicroBooNE Collaboration), J. Instrum. 12, P03011 (2017).

[2] C. Adams et al. (MicroBooNE Collaboration), Phys. Rev. D 99, 092001 (2019).

[3] A. Radovic, M. Williams, D. Rousseau, M. Kagan, D. Bonacorsi, A. Himmel, A. Aurisano, K. Terao, and T. Wongjirad, Nature (London) 560, 41 (2018).

[4] R. Acciarri et al. (MicroBooNE), J. Instrum. 12, P02017 (2017).

[5] M. Antonello et al. (MicroBooNE, LAr1-ND, and ICARUSWA104 Collaborations), arXiv:1503.01520.

[6] S. Amerio et al. (ICARUS Collaboration), Nucl. Instrum. Methods Phys. Res., Sect. A 527, 329 (2004).

[7] R. Acciarri et al. (DUNE Collaboration), arXiv:1601.02984.

[8] D. H. Koh, P. C. de Soux, L. Dominé, F. Drielsma, R. Itay, Q. Lin, K. Terao, K. V. Tsang, and T. Usher, arXiv:2007.03083.

[9] F. Drielsma, Q. Lin, P. C. de Soux, L. Dominé, R. Itay, D. H. Koh, B. J. Nelson, K. Terao, K. V. Tsang, and T. L. Usher, arXiv:2007.01335.
[10] F. Drielsma, K. Terao, L. Dominé, and D. H. Koh, arXiv:2102.01033.

[11] J. Marshall and M. Thomson, Eur. Phys. J. C 75, 439 (2015).

[12] R. Acciarri, C. Adams, R. An, J. Anthony, J. Asaadi, M. Auger, L. Bagby, S. Balasubramanian, B. Baller, C. Barnes et al., Eur. Phys. J. C 78, 82 (2018).

[13] K. He, G. Gkioxari, P. Dollár, and R. Girshick, in Proceedings of the IEEE International Conference on Computer Vision (Institute of Electrical and Electronics Engineers Inc., United States, 2017), pp. 2961-2969.

[14] R. Girshick, in Proceedings of the IEEE International Conference on Computer Vision (Institute of Electrical and Electronics Engineers Inc., United States, 2015), pp. 1440-1448.

[15] S. Ren, K. He, R. Girshick, and J. Sun, in Adv. Neural Inf. Process. Syst. (MIT Press, United States, 2015), pp. 91-99.

[16] W. Liu, D. Anguelov, D. Erhan, C. Szegedy, S. Reed, C.-Y. $\mathrm{Fu}$, and A. C. Berg, Lect. Notes Comput. Sci. 9905, 21 (2016). 
[17] L. Dominé and K. Terao (DeepLearnPhysics Collaboration), Phys. Rev. D 102, 012005 (2020).

[18] L. Dominé and K. Terao, Applying deep neural network techniques for LArTPC data reconstruction, 2018, https:// doi.org/10.5281/zenodo.1300713.

[19] V. V. Sochat, C. J. Prybol, and G. M. Kurtzer, PLoS One 12, 11 (2017).

[20] C. Adams, K. Terao, and T. Wongjirad, arXiv:2006 .01993 .

[21] B. Graham and L. van der Maaten, arXiv:1706.01307.

[22] B. Graham, M. Engelcke, and L. van der Maaten, in Proceedings of the IEEE Conference on Computer Vision and Pattern Recognition (Institute of Electrical and Electronics Engineers Inc., United States, 2018), pp. 9224-9232.

[23] O. Ronneberger, P. Fischer, and T. Brox, in International Conference on Medical Image Computing and ComputerAssisted Intervention (Springer, New York, 2015), Vol. 9351, pp. 234-241.

[24] M. Ester, H.-P. Kriegel, J. Sander, and X. Xu, in Proceedings of the Second International Conference on Knowledge Discovery and Data Mining, KDD'96 (AAAI Press, United States, 1996), pp. 226-231.

[25] Particle data group, atomic and nuclear properties of liquid argon (ar), retrieved 2017.

[26] L. Hubert and P. Arabie, J. Classif. 2, 193 (1985). 